\title{
Delayed Onset Parkinsonism in Alcoholic Patient Following ARDS: Alcoholism could be a Risk Factor
}

\author{
Patrick Desaive, Bernard Dan and Charles Kornreich ${ }^{*}$
}

\author{
Psychiatric Institute, CHU Brugmann, Place Van Gehuchten 4, 1020 Brussels, Belgium
}

\begin{abstract}
A case of delayed vascular parkinsonism resulting from an hypoxic insult is reported in a alcoholic patient. Hypoxic events are more frequent in alcoholic patients probably through a mechanism of increased lung vulnerability. When present, hypoxia has more severe neurological consequences in this population, occurring in a context of diminished cerebral blood flow. Alcoholism could be a risk factor for the occurrence of delayed onset parkinsonism following ARDS.
\end{abstract}

Keywords: Vascular Parkinsonism, ARDS, alcohol, apoptosis, delayed symptoms.

\section{CASE REPORT}

We report a 40-days delayed onset case of vascular parkinsonism resulting from an hypoxic insult (acute respiratory distress syndrome, ARDS). Parkinsonism following cerebral anoxia has been widely documented [14]. Our patient was alcoholic and this condition could have been a predisposing factor to his neurological outcome.

A 52 year-old man was admitted in coma following a medication suicide attempt with benzodiazepines. His psychiatric history included social phobia, long-term severe alcohol dependence, in remission for two months, and tobacco dependence.

Cardio-respiratory status was normal on admission. He went out of coma several hours later but had to be put on artificial ventilation due to acute pulmonary failure. Pulmonary failure was attributed to false deglutition and to inhalation pneumonia. He was extubated on day 10 and left the unit on day 12 , without any clinically apparent cognitive or motor deficits. Cardiac monitoring had been normal all along.

From day 45 on, the following neurological signs appeared progressively over 2 weeks, then remained stable: dysarthria, postural instability including unsteady gait, bradykinesia and reduced automatic movements. There was no tremor. Muscle tone was slightly increased with lower extremity predominance. There was no cogwheel phenomenon. Cognitive functions did not seem to be altered. Sphincter control remained intact. Electroencephalogram was normal. Brain magnetic resonance imaging showed moderate cerebellar atrophy, a common finding in heavy alcoholics [5] as well as patchy increase signal in the cortex (with a predominance in the temporal lobes), in the periventricular white matter and in the pons on T2-weighted sequences but no abnormal signal in the basal ganglia.

\footnotetext{
*Address correspondence to this author at the Psychiatric Institute, $\mathrm{CHU}$ Brugmann, Place Van Gehuchten 4, 1020 Brussels, Belgium; Tel: 3224772705; Fax: 3224772162; E-mail: ckornrei@ulb.ac.be
}

The patient was treated with levodopa and physical therapy. Slight clinical improvement was noted after 3 weeks. Six months after that episode, the patient still showed difficulties to walk and a rigidity.

This patient met the diagnostic criteria for vascular parkinsonism [6-9], i.e. co-existence of the clinical features described above and of cardio-vascular risk factors (obesity, tobaco consumption, sedentarity). Delayed-onset movement disorders are quite common after hypoxic or anoxic events such as carbon monoxyde poisonning, hypoglycemia and cardiac arrest $[1-3,10]$. In those cases, movement disorders are associated with encephalopathy, which commonly manifest with cognitive dysfunction, mutism or EEG abnormalities. None of these features were present in our patient.

The free interval between hypoxia and symptomatology is negatively linked to age, which presumably relates to the quality of the cerebral perfusion or metabolism [10].

Free radicals liberation linked to hypoxy provoke apoptosis and, secondary, clinical manifestations. Toxic effect of chronic alcohol intake on the brain is well known and don't seem to totally subside with abstinence [11]. This toxic effect can significantly interfere with brain metabolism and lead to increased apoptosis particularly in sensitive regions such as the striatum $[2,8]$.

Two mechanisms acting synergetically could account for the relationship between chronic alcohol intake and the vascular induced parkinsonism in our patient: Alcoholdependent individuals are both more susceptible to hypoxic events and to negative outcomes following hypoxic events. In studies involving critical care patients, chronic alcohol abuse increases the incidence of ARDS by nearly three-fold and is associated with more severe nonpulmonary organ dysfunction [12]. Decreased concentrations of gluthation in the epithelial lining fluid of the lung, which normally exercises a protective influence could predispose to such outcomes [12].

Heavy alcohol consumption is also associated with an increased risk of stroke [13]. A diminished cerebral blood 
flow might explain this association [13]. Animal models have shown that chronic alcohol consumption impairs cerebral vasoreactivity and thus may result in an increase in ischemic brain damage [14]. Chronic alcohol consumption in humans might therefore be a risk factor for ulterior brain damage when the brain is insulted.

Unfortunately, no secondary prevention strategies have been codified yet: hypothermia shows benefits in animal but

remains controversial in human [1]; Corticosteroids, aspirin and vasodilatators do not seem effective [15].

The trigger of the clinical course still remains unknow. It seems that hypoxia leads to secondary vascular lesions becoming apparent after resumption of activity [1]. From this point of view, rest seems to be protective.

Patients who have vascular risk factors, and among them alcoholic patients, should be monitorised carefuly after hypoxic or anoxic events even if complete recovery seems present.

\section{REFERENCES}

[1] Custodio CM, Basford JR. Delayed post-anoxic encephalopathy: a case report and literature review. Arch Phys Med Rehabil 2004; 85: 502-5.

[2] Sheth RD, Bodensteiner JB. Delayed post-anoxic encephalopathy: possible role for apoptosis. J Child Neurol 1998; 13: 347-8.

[3] Choi IS. Parkinson after carbone monoxyde poisoning. Eur Neurol 2002; 48: 30-3.
[4] Winikates J, Jankovic J. Clinical correlates of vascular parkinsonism. Arch Neurol 1999; 56: 98-102.

[5] Oscar-Berman M, Marinkovik K. Alcohol: effects on neurobehavioral functions and the brain. Neuropsychol Rev 2007; 17: 239-57.

[6] Zijlmans JC, Daniel SE, Hughues AJ, Révész T, Lees AJ. Clinicopathological investigation of vascular parkinsonism, including criteria for diagnosis. Mov Disord 2004; 19: 630-40.

[7] Demirkiran M, Bozdemir H, Sarica Y. Vascular parkinsonism: a distinct, heterogeneous clinical entity. Acta Neurol Scand 2001; 104: 63-7.

[8] Chang CM, Yu YL, Ng HK, Leung SY, Fong KY. Vascular pseudoparkinsonism. Acta Neurol Scand 1992; 86: 588-92.

[9] Tohgi H, Takahashi S, Abe T, Utsuqisawa K. Symptomatic characteristics of parkinsonism and the width of substantia nigra pars compacta on MRI according to ischemic changes in the putamen and cerebral white matter: implication for the diagnosis of vascular parkinsonism. Eur Neurol 2001; 46: 1-10.

[10] Choi IS, Cheon HY. Delayed movement disorders after carbon monoxide poisoning. Eur Neurol 1999; 42: 141-4.

[11] Neiman J. Alcohol as a risk factor for brain damage: neurologic aspects. Alcohol Clin Exp Res 1998; 22: 346S-351S.

[12] Moss M, Burnham EL. Chronic alcohol abuse, acute respiratory distress syndrome, and multiple organ dysfunction. Crit Care Med 2003; 31 (S): S207-S212.

[13] Reynolds K, Lewis B, Nolen JD, Kinney GL, Sathya B, He J. Alcohol consumption and risk of stroke. A meta-analysis. JAMA 2003; 289: 579-88.

[14] Sun H, Zhao H, Sharpe GM, Arrick DM, Mayhan WG. Effect of chronic alcohol consumption on brain damage following transient focal ischemia. Brain Res 2008; 1194: 73-80.

[15] Lee HB, Lyketsos CG. Delayed post-hypoxic leukoencephalopathy. Psychosomatics 2001; 42: 530-3. 